\title{
Asymptotics for the Tail Behavior of Total Claims for a Risk Model with Stochastic Returns
}

\author{
Yinghua Dong \\ College of Mathematics and Statistics, \\ Nanjing University of Information Science and Technology, \\ Nanjing, China \\ dongyinghua1@163.com
}

\begin{abstract}
This paper studies a two-dimensional risk model with stochastic returns, where the two kinds of claim sizes constitute a sequence of independent and identically distributed random vectors following a two-dimensional FGM distribution. When the two marginal distributions of the claim-size have dominated-varying tails, it gives an asymptotic formula for the tail probability of discounted total claims. The conclusion shows the dependence between two kinds of claims has an effect on the asymptotic behavior of the tail of discounted total claims.
\end{abstract}

Keywords-Asymptotic behavior; nonstandard risk model; discounted total claims; stochastic return

\section{INTRODUCTION}

In this paper, we consider a nonstandard renewal risk model with stochastic return, in which an insurance company simultaneously operates two different kinds of businesses. For any $t \geq 0$, the two lines of surplus processes of the insurance company are denoted by

$$
\begin{aligned}
& U_{1}(t)=x e^{R_{1}(t)}+c_{1} \int_{0}^{t} e^{R_{1}(t)-R_{1}(s)} d s-\sum_{i=1}^{M(t)} X_{i} e^{R_{1}(t)-R_{1}\left(\tau_{i}\right)}, \\
& U_{2}(t)=x e^{R_{2}(t)}+c_{2} \int_{0}^{t} e^{R_{2}(t)-R_{2}(s)} d s-\sum_{j=1}^{N(t)} Y_{j} e^{R_{2}(t)-R_{2}\left(\eta_{j}\right)},
\end{aligned}
$$

respectively. The above surplus processes satisfy the following assumptions: the claim sizes $\left\{(\mathrm{X}, \mathrm{Y}),\left(X_{i}, Y_{i}\right), i \geq 1\right\}$ form a sequence of independent, identically distributed and nonnegative random vectors with generic vector $(X, Y)$ whose marginal distributions are written by $F$ and $G,(x, y)$ denote the initial capital vector, and $\left(c_{1}, c_{2}\right)$ is the premium vector in a unit time. The price process of the investment portfolios for the two kinds of insurance businesses are described as geometric Lévy processes $\left\{e^{R_{1}(t)}, t \geq 0\right\}$ and $\left\{e^{R_{2}(t)}, t \geq 0\right\}$, respectively, where $\left\{R_{1}(t), t \geq 0\right\}$ and $\left\{R_{2}(t), t \geq 0\right\}$ are two independent Lévy processes. $\{M(t), t \geq 0\}$ and $\{N(t), t \geq 0\}$ represent the arrival processes with renewal functions $\lambda_{1}(t)$ and $\lambda_{2}(t)$, respectively. We suppose that $\left\{(X, Y),\left(X_{i}, Y_{i}\right), i \geq 1\right\}$, $\left\{R_{1}(t), t \geq 0\right\},\left\{R_{2}(t), t \geq 0\right\},\{M(t), t \geq 0\}$ and $\{N(t), t \geq 0\}$

This research was supported by Project Funded by China Postdoctora (No.2016M591885) and Jiangsu Planned Projects for Postdoctoral Research Funds (No. 1501053A) are mutually independent. Further, we can easily prove that

$$
\lambda_{1}(t)=\sum_{i=1}^{\infty} P\left(\tau_{i} \leq t\right) \text { and } \lambda_{2}(t)=\sum_{j=1}^{\infty} P\left(\eta_{j} \leq t\right) .
$$

Throughout this paper, we suppose that $(X, Y)$ follows a two-dimensional Sarmanov distribution of the following form:

$$
P(X \in d x, Y \in d y)=(1+\theta) F(d u) G(d v),
$$

where $\theta \in(-1,1]$ is a real number.

We suppose that the two Lévy processes $\left\{R_{1}(t), t \geq 0\right\}$ and $\left\{R_{2}(t), t \geq 0\right\}$ are right continuous with left limits. Assume that $E R_{1}(1)>0$ and $E R_{2}(1)>0$, so that both $R_{1}(t)$ and $R_{2}(t)$ tend to infinity as $t \rightarrow \infty$. The Laplace exponents for the Lévy processes $\left\{R_{1}(t), t \geq 0\right\}$ and $\left\{R_{2}(t), t \geq 0\right\}$ are defined as

$\phi_{i}(\mathrm{z})=\log E e^{-z R_{i}(1)}, \mathrm{z} \geq 0, i=1,2$.

If $\phi_{i}(z)$ finite, then for any $t \geq 0, E e^{-z R_{i}(t)}=e^{t \phi_{i}(z)}<\infty$,

see Proposition 3.14 of [1] for details.

In the recent years, the one-dimensional renewal risk model with stochastic return has been widely studied. We refer the reader to [2] and [3]. In addition, few articles considered a two-dimensional risk model with stochastic returns. For instance,[4] considered a two-dimensional nonstandard renewal risk model with two correlated classes of insurance businesses and risk investments, when the correlation comes from a common shock, the claim sizes of the same kind business are pairwise quasi-asymptotically independent, but different kinds of claim sizes are independent, the authors gave a uniformly asymptotic formula.

In the present paper, we study a two-dimensional nonstandard renewal risk model, where the claim sizes of different kinds of insurance businesses form a sequence of independent, identically distributed and random vectors, and the dependence structure between different insurance businesses is depicted by a FGM distribution. When the marginal distributions belong to the intersection of the dominated variation class and the class of long-tailed distribution, we obtain an asymptotic formula of the tail probability of discounted total claims. 


\section{PRELIMINARIES AND MAIN RESULTS}

The paper is related to heavy-tailed distributions, so we first introduce important subclasses of heavy-tailed distributions. We can refer the reader to [5]. Let $X$ be a random variable with distribution $F$. We assume that $\bar{F}(x)>0$ for all $x>0$.

A distribution $F$ is said to belong to the class of the longtailed distributions $L$, if for any $u>0$,

$$
\lim _{x \rightarrow \infty} \frac{\bar{F}(x+u)}{\bar{F}(x)}=1 .
$$

We call a distribution $F$ to belong to the dominated variation class $D$, if for any $v>0$, the relation

$$
\lim _{x \rightarrow \infty} \frac{\bar{F}(v x)}{\bar{F}(x)}=1 .
$$

For any distribution $F$ on $[0, \infty)$, denote

$$
J_{F}^{+}=-\lim _{y \rightarrow \infty} \frac{\log \overline{F_{*}}(y)}{\log y} \text { and } J_{F}^{-}=-\lim _{y \rightarrow \infty} \frac{\log \overline{F^{*}}(y)}{\log y}
$$

They are called the upper and lower Matuszewska indices, respectively. if $F \in D$, then $0 \leq J_{F}^{-} \leq J_{F}^{+}<\infty$.

Hereafter, all limit relation for $\min \{x, y) \rightarrow \infty$ unless stated otherwise. For two positive functions $a(x, y)$ and $b(x, y)$, write $a(x, y) \sim b(x, y)$, if

$$
\lim _{\min (x, y) \rightarrow \infty} \frac{a(x, y)}{b(x, y)}=1 .
$$

Now we present the main result of this paper.

Theorem 1 Consider the above renewal risk model. Let $\left\{(\mathrm{X}, \mathrm{Y}),\left(X_{i}, Y_{i}\right), i \geq 1\right\}$ is a sequence of independent, identically distributed random vectors following a common twodimensional FGM distribution of the form (1). Suppose that the distributions of $X$ and $Y$ satisfy $F \in D \cap L$ and $G \in D \cap L$. If the Laplace exponents $\phi_{i}\left(\beta_{i}\right)<0, i=1,2$, for some $\beta_{1}>J_{F}^{+}$ and $\beta_{2}>J_{G}^{+}$, then for any fixed $T>0$ satisfy $\lambda_{1}(T)>0$ and $\lambda_{2}(T)>0$, if $\theta \in[-1,0]$, then

$$
\begin{gathered}
P\left(\sum_{i=1}^{M(T)} X_{i} e^{-R_{1}\left(\tau_{i}\right)}>x, \quad \sum_{j=1}^{N(T)} Y_{j} e^{-R_{2}\left(\eta_{j}\right)}>y\right) \\
\sim \int_{0}^{T} \int_{0}^{T} P\left(X e^{-R_{1}(u)}>x\right) P\left(Y e^{-R_{2}(v)}>y\right) d \lambda_{1}(u) d \lambda_{2}(v)+ \\
\theta \int_{0}^{T} \int_{0}^{T} P\left(X e^{-R_{1}(u)}>x\right) P\left(Y e^{-R_{2}(v)}>y\right) d P\left(\tau_{i} \leq u\right) d P\left(\eta_{i} \leq v\right) .
\end{gathered}
$$

\section{SOME LEMMAS}

Before proving the conclusion, we give some necessary lemmas.

The following lemma is from [6].
Lemma 3.1If $F \in L$, then there exists a positive function

$l(x)$ satisfying $0<l(x)<\infty$ and $l(x) / x \rightarrow 0$ such that

$$
\lim _{x \rightarrow \infty} \frac{\bar{F}(x+l(x))}{\bar{F}(x)}=1 .
$$

The following lemma is due to Proposition 2.2.1 of [5] and Lemma 3.5 of [7].

Lemma 3.2For a distribution $F$ on $[0, \infty)$, the following inequalities hold:

(1) If $F \in D$, then for any $\alpha<J_{F}^{-}$and $\beta<J_{F}^{+}$, there are

Positive numbers $C_{i}$ and $D_{i}, i=1,2$, such that

$$
\frac{\bar{F}(y)}{\bar{F}(x)} \geq C_{1}\left(\frac{x}{y}\right)^{\alpha} \text { for all } x \geq y \geq D_{1}
$$

and

$$
\frac{\bar{F}(y)}{\bar{F}(x)} \leq C_{2}\left(\frac{x}{y}\right)^{\beta} \text { for all } x \geq y \geq D_{2}
$$

(2) If $F \in D$, then

$$
x^{\beta} \bar{F}(x) \rightarrow \infty \text {, as } x \rightarrow \infty \text {. }
$$

The lemma below is due to Wang and Tang [8].

Lemma 3.3Let $X$ and $\xi$ be two independent random variables, where $X$ is distributed by $F \in D \cap L$ and $\xi$ is nonnegative and non-degenerate at 0 random variables satisfying $E \xi^{p}<\infty$ for some $p>J_{F}^{+}$. Then the distribution of $\xi X$ belongs to the class $D \cap L$ and

$$
0<\liminf _{x \rightarrow \infty} \frac{P(\xi X>x)}{P(X>x)}<\limsup _{x \rightarrow \infty} \frac{P(\xi X>x)}{P(X>x)}<\infty .
$$

The lemma below is due to [9].

Lemma 3.4Let $\left\{X_{i}, 1 \leq i \leq n\right\}$ be a sequence of independent random variables with common distribution $F \in D \cap L$, $\left\{\xi_{i}, 1 \leq i \leq n\right\}$ is a sequence of nonnegative and non-degenerate at 0 random variables satisfying $E \xi_{i}^{p}<\infty$ for some $p>J_{F}^{+}$and independent of $\left\{X_{i}, 1 \leq i \leq n\right\}$. Then

$$
\lim _{\min (x, y) \rightarrow \infty} \frac{P\left(\xi_{i} X_{i}>x, \xi_{j} X_{j}>y\right)}{P\left(\xi_{i} X_{i}>x\right)}=0
$$

holds for all $1 \leq i \neq j \leq n$.

The following lemma plays an important in proving the main result.

Lemma 3.5Let $F$ and $G$ follow a FGM distribution (1). Suppose that the distributions of $X$ and $Y$ satisfy $F \in D \cap L$ and $G \in D \cap L$. If the Laplace exponents $\phi_{i}\left(\beta_{i}\right)<0, i=1,2$, for some $\beta_{1}>J_{F}^{+}$and $\beta_{2}>J_{G}^{+}$, then it holds that 
$P\left(X e^{-R_{1}(u)}>x, Y e^{-R_{2}(v)}>y\right)$

$\sim(1+\theta) P\left(X e^{-R_{1}(u)}>x\right) P\left(Y e^{-R_{2}(v)}>y\right)$.

Proof. By (1), we have

$P(X>x, Y>y)=(1+\theta) P(X>x) P(Y>y)$

Choose some $0<\varepsilon<1$. We can see that

$$
\begin{aligned}
& P\left(X e^{-R_{1}(u)}>x, Y e^{-R_{2}(v)}>y\right) \\
= & P\left(X e^{-R_{1}(u)}>x, Y e^{-R_{2}(v)}>y, e^{-R_{1}(u)} \leq x^{1-\varepsilon}, e^{-R_{2}(v)}>y^{1-\varepsilon}\right) \\
= & P\left(X e^{-R_{1}(u)}>x, Y e^{-R_{2}(v)}>y, e^{-R_{1}(u)}>x^{1-\varepsilon}, e^{-R_{2}(v)}>y^{1-\varepsilon}\right) \\
= & P\left(X e^{-R_{1}(u)}>x, Y e^{-R_{2}(v)}>y, e^{-R_{1}(u)}>x^{1-\varepsilon}, e^{-R_{2}(v)}>y^{1-\varepsilon}\right) \\
= & P\left(X e^{-R_{1}(u)}>x, Y e^{-R_{2}(v)}>y, e^{-R_{1}(u)} \leq x^{1-\varepsilon}, e^{-R_{2}(v)} \leq y^{1-\varepsilon}\right) \\
\equiv & \left.I_{1} x, y\right)+I_{2}(x, y)+I_{3}(x, y)+I_{4}(x, y)
\end{aligned}
$$

For $I_{1}(x, y)$, by (1), we

$$
I_{1}(x, y) \leq P\left(X e^{-R_{1}(u)}>x^{\prime}\right) P\left(e^{-R_{2}(v)}>y^{1-\varepsilon}\right) .
$$

For some $p>J_{F}^{+}$and sufficiently small and positive $\varepsilon$, by Markov inequality, we have

$$
\frac{P\left(e^{-R_{2}(v)}>y\right)}{\bar{G}(y)} \leq \frac{E e^{-p R_{2}(v)}}{y^{p(1-\varepsilon)} \bar{G}(y)}=\frac{e^{v \phi_{2}(p)}}{y^{p(1-\varepsilon)} \bar{G}(y)} \rightarrow 0 \text {, as } y \rightarrow \infty .
$$

According to Lemma3.3, we arrive at

$$
I_{1}(x, y)=o(1) P\left(X e^{-R_{1}(u)}>x\right) P\left(Y e^{-R_{2}(v)}>y\right) .
$$

Similarly as above,

$$
I_{k}(x, y)=o(1) P\left(X e^{-R_{1}(u)}>x\right) P\left(Y e^{-R_{2}(v)}>y\right), k=2,3 .
$$

Finally, we discuss $I_{4}(x, y)$. By (1), (3) and (4), we have

$$
\begin{aligned}
& I_{4}(x, y) \sim(1+\theta) \times \\
& \int_{0}^{x^{1-\varepsilon}} \int_{0}^{y^{1-\varepsilon}} P\left(X>\frac{x}{z_{1}}\right) P\left(Y>\frac{y}{z_{2}}\right) P\left(e^{-R_{1}(u)} \in d z_{1}\right) P\left(e^{-R_{2}(v)} \in d z_{2}\right) \\
& \sim(1+\theta) P\left(X e^{-R_{1}(u)}>x\right) P\left(Y e^{-R_{2}(v)}>y\right)
\end{aligned}
$$

Substituting (3), (4), (5) into (2), we prove Lemma 3.5.

Lemma 3.6Let $\left\{(\mathrm{X}, \mathrm{Y}),\left(X_{i}, Y_{i}\right), i \geq 1\right\}$ is a sequence of independent, identically distributed random vectors following a common two-dimensional FGM distribution of the form (1). Suppose that the distributions of $X$ and $Y$ satisfy $F \in D \cap L$ and $G \in D \cap L$, then for any fixed $m \geq 1$ and $n \geq 1$, it holds uniformly for all $0 \leq s_{i} \leq T$ and $0 \leq t_{i} \leq T$,

$$
\begin{array}{r}
P\left(\sum_{i=1}^{m} X_{i} e^{-R_{1}\left(s_{i}\right)}>x, \sum_{j=1}^{n} Y_{j} e^{-R_{2}\left(t_{j}\right)}>y\right) \\
\sim \sum_{i=1}^{m} \sum_{j=1}^{n} P\left(X e^{-R_{1}\left(s_{i}\right)}>x, Y e^{-R_{2}\left(t_{j}\right)}>y\right) .
\end{array}
$$

Proof. With loss of generality, we assume that $m>n$. Denote

$$
E_{1}=\bigcup_{i=1}^{m}\left(X_{i} e^{-R_{1}\left(s_{i}\right)}>x-h_{1}(x)\right) E_{2}=\bigcup_{i=1}^{m}\left(Y_{j} e^{-R_{2}\left(t_{j}\right)}>y-h_{2}(y)\right) .
$$

Where $P\left(X_{i} e^{-R_{1}\left(s_{i}\right)}>x-h_{1}(x)\right) \sim P\left(X_{i} e^{-R_{1}\left(s_{i}\right)}>x\right)$, and

$$
P\left(Y_{j} e^{-R_{2}\left(t_{j}\right)}>y-h_{2}(y)\right) \sim P\left(Y_{j} e^{-R_{2}\left(t_{j}\right)}>y\right) .
$$

Intersect the event

$$
P\left(\sum_{i=1}^{m} X_{i} e^{-R_{1}\left(s_{i}\right)}>x, \sum_{j=1}^{n} Y_{j} e^{-R_{2}\left(t_{j}\right)}>y\right)
$$

With $E_{1} E_{2}, E_{2}^{c}$ and $E_{1}^{c}$, respectively, and denote the corresponding probabilities by $J_{1}(x, y), J_{2}(x, y)$ and $J_{3}(x, y)$.

For $J_{1}(x, y)$, we can see that

$$
\begin{aligned}
& J_{1}(x, y) \\
& \leq \sum_{i=1}^{m} \sum_{j=1}^{n} P\left(X e^{-R_{1}\left(s_{i}\right)}>x-h_{1}(x), Y e^{-R_{2}\left(t_{j}\right)}>y-h_{2}(y)\right) \\
& \sim \sum_{i=1}^{m} \sum_{j=1, j \neq i}^{n} P\left(X e^{-R_{1}\left(s_{i}\right)}>x\right) P\left(Y e^{-R_{2}\left(t_{j}\right)}>y\right) \\
& +(1+\theta) \sum_{i=1}^{m} P\left(X e^{-R_{1}\left(s_{i}\right)}>x\right) P\left(Y e^{-R_{2}\left(t_{j}\right)}>y\right) \\
& \sim \sum_{i=1}^{m} \sum_{j=1}^{n} P\left(X e^{-R_{1}\left(s_{i}\right)}>x, Y e^{-R_{2}\left(t_{j}\right)}>y\right) .
\end{aligned}
$$

Next we deal with $J_{2}(x, y)$.

$$
J_{2}(x, y)
$$

$$
\begin{aligned}
& \leq \sum_{i=1}^{m} \sum_{k=1}^{n} \sum_{j=1, j \neq k}^{n} P\left(X_{i} e^{-R_{1}\left(s_{i}\right)}>x / n, Y_{j} e^{-R_{2}\left(t_{j}\right)}>l(y) / n, Y_{k} e^{-R_{2}\left(t_{k}\right)}>y / n\right) \\
& +\sum_{i=1}^{m} \sum_{j=1, j \neq i}^{n} P\left(X_{i} e^{-R_{1}\left(s_{i}\right)}>x / n, Y_{j} e^{-R_{2}\left(t_{j}\right)}>l(y) / n, Y_{i} e^{-R_{2}\left(t_{k}\right)}>y / n\right) \\
& +\sum_{i=1}^{m} \sum_{k=1, k \neq i}^{n} P\left(X_{i} e^{-R_{1}\left(s_{i}\right)}>x / n, Y_{i} e^{-R_{2}\left(t_{j}\right)}>l(y) / n, Y_{k} e^{-R_{2}\left(t_{k}\right)}>y / n\right) \\
& \equiv J_{21}(x, y)+J_{22}(x, y)+J_{23}(x, y) .
\end{aligned}
$$

For $J_{21}(x, y)$, by Lemma 3.3 and Lemma 3.4, we have

$$
\begin{aligned}
& J_{21}(x, y) \leq \sum_{i=1}^{m} P\left(X_{i} e^{-R_{1}\left(s_{i}\right)}>x / n\right) \\
& \quad \times \sum_{k=1}^{n} \sum_{j=1, j \neq i}^{n} P\left(Y_{j} e^{-R_{2}\left(t_{j}\right)}>l(y) / n, Y_{k} e^{-R_{2}\left(t_{k}\right)}>y / n\right) \\
& =o(1) \sum_{i=1}^{m} \sum_{j=1}^{n} P\left(X e^{-R_{1}\left(s_{i}\right)}>x, Y e^{-R_{2}\left(t_{j}\right)}>y\right) .
\end{aligned}
$$

Following the above proof with a modification, we have 


$$
\begin{gathered}
J_{2 k}=o(1) \sum_{i=1}^{m} \sum_{j=1}^{n} P\left(X e^{-R_{1}\left(s_{i}\right)}>x, Y e^{-R_{2}\left(t_{j}\right)}>y\right), k=2,3 . \\
J_{2}=o(1) \sum_{i=1}^{m} \sum_{j=1}^{n} P\left(X e^{-R_{1}\left(s_{i}\right)}>x, Y e^{-R_{2}\left(t_{j}\right)}>y\right) .
\end{gathered}
$$

In the same way,

$$
J_{3}=o(1) \sum_{i=1}^{m} \sum_{j=1}^{n} P\left(X e^{-R_{1}\left(s_{i}\right)}>x, Y e^{-R_{2}\left(t_{j}\right)}>y\right) .
$$

By (7), (8) and (9) , we arrive at the asymptotic upper bound of (6). Then we discuss the asymptotic low bound of (6).

$$
\begin{aligned}
& P\left(\sum_{i=1}^{m} X_{i} e^{-R_{1}\left(s_{i}\right)}>x, \sum_{j=1}^{n} Y_{j} e^{-R_{2}\left(t_{j}\right)}>y\right) \\
& \geq P\left(\bigcup_{i=1}^{m}\left(X_{i} e^{-R_{1}\left(s_{i}\right)}>x\right), \bigcup_{j=1}^{n}\left(Y_{j} e^{-R_{2}\left(t_{j}\right)}>y\right)\right) \\
& \geq \sum_{i=1}^{m} \sum_{j=1}^{n} P\left(X_{i} e^{-R_{1}\left(s_{i}\right)}>x, Y_{j} e^{-R_{2}\left(t_{j}\right)}>y\right)- \\
& \sum_{i=1}^{m} \sum_{k=1}^{n} \sum_{j=1, j \neq k}^{n} P\left(X_{i} e^{-R_{1}\left(s_{i}\right)}>x, Y_{k} e^{-R_{2}\left(t_{k}\right)}>y, Y_{j} e^{-R_{2}\left(t_{j}\right)}>y\right)- \\
& \sum_{i=1}^{m} \sum_{j=1}^{n} \sum_{k=1, k \neq i}^{n} P\left(X_{i} e^{-R_{1}\left(s_{i}\right)}>x, X_{k} e^{-R_{1}\left(s_{k}\right)}>y, Y_{j} e^{-R_{2}\left(t_{j}\right)}>y\right) .
\end{aligned}
$$

Similarly as in $J_{21}(x, y)$, we can get that the second and third terms on the right-hand side of (10) is asymptotically negligible compared with the first one. We complete the proof.

\section{PROOF OF THE MAIN RESUlT}

Proof. For some fixed positive integer $M$, it holds that

$$
\begin{gathered}
P\left(\sum_{i=1}^{M(T)} X_{i} e^{-R_{1}\left(\tau_{i}\right)}>x, \sum_{j=1}^{N(T)} Y_{j} e^{-R_{2}\left(\eta_{j}\right)}>y\right) \\
=\sum_{m=1}^{\infty} \sum_{n=1}^{\infty} P\left(\sum_{i=1}^{m} X_{i} e^{-R_{1}\left(\tau_{i}\right)}>x, \sum_{j=1}^{n} Y_{j} e^{-R_{2}\left(\eta_{j}\right)}>y, M(T)=m, N(T)=n\right) \\
=\left(\sum_{m=1}^{M} \sum_{n=1}^{M}+\sum_{m=1}^{M} \sum_{n=M+1}^{\infty}+\sum_{m=M+1}^{\infty} \sum_{n=1}^{M}+\sum_{m=M+1}^{\infty} \sum_{n=M+1}^{\infty}\right) \\
P\left(\sum_{i=1}^{m} X_{i} e^{-R_{1}\left(\tau_{i}\right)}>x, \sum_{j=1}^{n} Y_{j} e^{-R_{2}\left(\eta_{j}\right)}>y, M(T)=m, N(T)=n\right) \\
\equiv K_{1}(x, y ; T)+K_{2}(x, y ; T)+K_{3}(x, y ; T)+K_{4}(x, y ; T) .
\end{gathered}
$$

We first consider $K_{1}(x, y ; T)$. By Lemma 3.6, it follows

$$
\begin{aligned}
& K_{1}(x, y ; T) \\
& \sim \sum_{m=1}^{M} \sum_{n=1}^{M} \sum_{i=1}^{m} \sum_{j=1}^{n} P\left(X_{i} e^{-R_{1}\left(\tau_{i}\right)}>x, Y_{j} e^{-R_{2}\left(\eta_{j}\right)}>y, M(t)=m, N(t)=n\right)
\end{aligned}
$$

$$
\begin{gathered}
\sim \int_{0}^{T} \int_{0}^{T} P\left(X e^{-R_{1}(u)}>x\right) P\left(Y e^{-R_{2}(v)}>y\right) d \lambda_{1}(u) d \lambda_{2}(v)+ \\
\theta \int_{0}^{T} \int_{0}^{T} P\left(X e^{-R_{1}(u)}>x\right) P\left(Y e^{-R_{2}(v)}>y\right) d P\left(\tau_{i} \leq u\right) d P\left(\eta_{i} \leq v\right) .
\end{gathered}
$$

Next we handle $K_{2}(x, y ; T)$. By Lemma 3.2,

$$
\begin{aligned}
& K_{2}(x, y ; T) \\
\leq & (1+|\theta|) P\left(X e^{-\inf _{0 \leq u \leq T} R_{1}(u)}>x\right) \times P\left(Y e^{-\inf _{0 \leq v \leq T} R_{2}(v)}>y\right) \\
& E(M(T) N(T))^{p+1} I_{(N(T) \geq M) .}
\end{aligned}
$$$$
\text { As } M \rightarrow \infty \text { and } \min (x, y) \rightarrow \infty \text {, }
$$$$
K_{2}(x, y ; T)=o(1) \int_{0}^{T} \int_{0}^{T} P\left(X e^{-R_{1}(u)}>x\right) P\left(Y e^{-R_{2}(v)}>y\right) d \lambda_{1}(u) d \lambda_{2}(v) .
$$

Similarly as above, for $\mathrm{i}=3,4$,

$$
K_{i}(x, y ; T)=o(1) \int_{0}^{T} \int_{0}^{T} P\left(X e^{-R_{1}(u)}>x\right) P\left(Y e^{-R_{2}(v)}>y\right) d \lambda_{1}(u) d \lambda_{2}(v) .
$$

\section{CONCLUSION}

We consider a nonstandard two-dimensional renewal risk model and aim to study the tail probability of discounted total claims. By using the method of randomly weighted sums, we derived the tail behavior of discounted total claims. The result shows the dependence between two kinds of claims has an effect on the tail probability of discounted total claims.

\section{REFERENCES}

[1] R. Cont and P. Tankov, Financial Modelling with Jump Processes. Chapman \& Hall/CRC: London, 2004.

[2] Q. Tang, G. Tang and K.C. Yuen, Uniform tail asymptotics for the stochastic present value of aggregate claims in the renewal risk model, Insurance Math. Econom. 2010, 46(2), 362-370.

[3] Y. Dong and Y. Wang, Uniform estimates for ruin probabilities in the renewal risk model with upper-tail independent claims and premiums, J. Ind. Manag. Optim. 2011, 7(4), 849-874.

[4] K. A. Fu and C.Y. A. Ng, Uniform tail asymptotics for the sum of two correlated classes with stochastic returns and dependent heavy tails, Stochastic Models, 30,197-215.

[5] N. H. Bingham, C. M. Goldie and J. L. Teugels, Regular Variation. 1987, Cambridge University Press, Cambridge.

[6] S. Foss, D. Korshunov and S. Zachary, An Introduction to Heavy-tailed and Subexponential Distributions, 2nd ed., Springer Series in Operation Research and Financial Engineerin, New York.

[7] Q. Tang and G. Tsitsiashvili, Precise estimates for the ruin probability in finite horizon in a discrete-time model with heavy-tailed insurance nd financial risks, stochatic Process. Appl.2003, 108, 299-325.

[8] D. Wang and Q. Tang, Tail probabilities of random variables with dominated variation, 2006, Stochastic Models, 22, 253-272.

[9] H. Yang. Asymptotic tail probability of randomly weighted sum of dependent random variables with dominated variation, Acta Math. Appl. Sin-E,2011, 27(3), 277-280. 